\title{
Effective Coulomb interaction in transition metals from constrained random-phase approximation
}

\author{
Ersoy Şaşıŏlu, ${ }^{*}$ Christoph Friedrich, and Stefan Blügel \\ Peter Grünberg Institut and Institute for Advanced Simulation, Forschungszentrum Jülich and JARA, D-52425 Jülich, Germany
}

(Received 18 January 2011; revised manuscript received 26 February 2011; published 25 March 2011)

\begin{abstract}
The effective on-site Coulomb interaction (Hubbard $U$ ) between localized $d$ electrons in $3 d, 4 d$, and $5 d$ transition metals is calculated employing a parameter-free realization of the constrained random-phase approximation using Wannier functions within the full-potential linearized augmented-plane-wave method. The $U$ values lie between 1.5 and $5.7 \mathrm{eV}$ and depend on the crystal structure, spin polarization, $d$ electron number, and $d$ orbital filling. On the basis of the calculated $U$ parameters, we discuss the strength of the electronic correlations and the instability of the paramagnetic state toward the ferromagnetic one for $3 d$ metals.
\end{abstract}

DOI: 10.1103/PhysRevB.83.121101

PACS number(s): 71.15.-m, 71.28.+d, 71.10.Fd

Density functional theory (DFT) within the local-density approximation $(\mathrm{LDA})^{1}$ is a reliable method for calculating ground-state properties of solids with weak electronic correlations, i.e., for $U / W<1$, where $U$ is the effective on-site Coulomb interaction between localized electrons (Hubbard $U$ ) and $W$ is the bandwidth. However, the LDA often fails to describe systems with intermediate $(U / W \sim 1)$ and strong $(U / W>1)$ electron correlations, such as transition-metal oxides, rare earths, Kondo systems, etc. Properties of these materials are usually calculated with phenomenological manybody Hamiltonians such as the Hubbard ${ }^{2}$ or the Anderson impurity model. ${ }^{3}$ However, in these models the Coulomb and also the one-particle hopping matrix elements are typically empirical parameters that are determined such that the employed model reproduces experimental results of interest.

For a long time, DFT-LDA and many-body model Hamiltonian methods have been separate and complementary approaches. This has drastically changed with the advent of the dynamical mean-field theory (DMFT) ${ }^{4}$ which merged with the LDA to become a novel computational method referred to as LDA + DMFT, ${ }^{5}$ which developed into a modern many-body approach for treating correlated electron materials. In retrospect, the so-called LDA $+U$ method, ${ }^{6}$ an early attempt to correct the LDA functional by introducing a simple mean-field-like Hubbard $U$ term for localized $d$ or $f$ states, and today routinely applied to a broad spectrum of systems, can be regarded as its static limit. Both LDA $+U$ and LDA + DMFT as well as other approaches not mentioned here rely on the Hubbard $U$ as an additional parameter. Frequently, the exact value of $U$ is unknown, which impedes the predictive power of these approaches.

The problem of calculating the parameter-free Hubbard $U$ for transition metals (TMs), i.e., from first principles, has been addressed by several authors. ${ }^{7-11}$ A number of different approaches have been proposed. Among them, the constrained local-density approximation (cLDA) ${ }^{9}$ is the most popular. However, the cLDA is known to give unreasonably large $U$ values for late TMs due to difficulties in compensating for the self-screening error of the localized electrons. ${ }^{10}$ Furthermore, the frequency dependence of $U$ is unattainable. On the other hand, the constrained random-phase approximation (cRPA), though numerically much more demanding, does not suffer from these difficulties. In contrast to the cLDA, it also allows access to individual Coulomb matrix elements, e.g., on-site, off-site, intra-orbital, interorbital, and exchange.

The aim of this Rapid Communication is to present a systematic study of the effective on-site Coulomb interaction (Hubbard $U$ ) between localized $d$ electrons in TMs determined by means of first-principles calculations. Previous cRPA studies of $U$ in TMs have focused only on the nonmagnetic (NM) state of the $3 d$ series, and the results appeared to be strongly dependent on the parameters used in the cRPA schemes. ${ }^{10,11}$ In the present work, we propose an alternative simple parameter-free cRPA approach within the full-potential linearized augmented-plane-wave (FLAPW) method using maximally localized Wannier functions (MLWFs). ${ }^{12}$ In addition to NM and magnetic states of the $3 d$ series, we consider $4 d$ and $5 d$ elements in the Periodic Table. We discuss the strength of the electronic correlations and the instability of the paramagnetic state toward the ferromagnetic one for $3 d$ TMs on the basis of the calculated Hubbard $U$ parameters.

The basic idea of the cRPA is to define an effective interaction $U$ between the localized $d$ electrons by restricting the screening processes to those that are not explicitly treated in the effective model Hamiltonian. ${ }^{10}$ To this end, we divide the full polarization matrix $P=P_{d}+P_{r}$, where $P_{d}$ includes only $d$ - $d$ transitions and $P_{r}$ is the remainder. Then, the frequency-dependent effective Coulomb interaction is given schematically by the matrix equation $U(\omega)=[1-$ $\left.v P_{r}(\omega)\right]^{-1} v$, where $v$ is the bare Coulomb interaction and $U(\omega)$ is related to the fully screened interaction by $\tilde{U}(\omega)=[1-$ $\left.U(\omega) P_{d}(\omega)\right]^{-1} U(\omega)$. The static limit of the average diagonal matrix element of $U(\omega \rightarrow 0)$ represented in a local basis can be regarded as the Hubbard $U$ parameter. ${ }^{10}$

Although the cRPA is a general approach, its application to materials with entangled bands is not straightforward. In these materials, the localized $d$ states that span the model subspace mix with extended $s$ and $p$ states, and there is no unique identification of the $d$ - $d$ transitions for constructing $P_{d}$. Several procedures have been proposed in the literature to overcome this problem. Aryasetiawan et al. ${ }^{10}$ suggested using an energy window or a range of band indices to define the $d$ subspace. However, the results depended strongly on the chosen window or band indices. An alternative approach, ${ }^{11}$ in which the hybridization of the $d$ states was switched off, was not burdened by additional parameters, but the $U$ values 
turned out to be unphysically large for materials with strong $s p$ - $d$ mixing, e.g., early TMs, and the unphysical suppression of hybridization is unsatisfactory.

In the present work, we propose a parameter-free procedure in which $P_{d}$ is directly constructed from the definition of the $d$ subspace. The latter is spanned by a set of MLWFs $w_{n \mathbf{R}}^{\alpha}(\mathbf{r})=\frac{1}{N} \sum_{\mathbf{k}, m} T_{\mathbf{R}, m n}^{\alpha}(\mathbf{k}) \varphi_{\mathbf{k} m}^{\alpha}(\mathbf{r})$, where $N$ is the number of $\mathbf{k}$ points, $T_{\mathbf{R}, m n}^{\alpha}(\mathbf{k})$ is the unitary transformation matrix, $\varphi_{\mathbf{k} m}^{\alpha}(\mathbf{r})$ are single-particle Bloch states of spin $\alpha$ and band index $m$, and $\mathbf{R}$ is the atomic position vector in the unit cell. We now define $P_{d}$ as the polarization function that is generated by all transitions that take place within the $d$ subspace. To determine with what probability this applies to a given transition between extended Bloch eigenstates $\varphi_{\mathbf{k} m}^{\alpha} \rightarrow \varphi_{\mathbf{k}+\mathbf{q} m^{\prime}}^{\alpha}$, which may be mixtures of $s p$ and $d$ states, we multiply the probability that the electron resides in the $d$ subspace before the transition $p_{\mathbf{k} m}^{\alpha}=\sum_{\mathbf{R}, n}\left|T_{\mathbf{R}, m n}^{\alpha}(\mathbf{k})\right|^{2}$ with the corresponding probability after the transition, and we obtain $p_{\mathbf{k} m}^{\alpha} p_{\mathbf{k}+\mathbf{q} m^{\prime}}^{\alpha}$ as the probability for the transition itself. Thus, $P_{d}$ is constructed from summing over all transitions in the Lehmann representation multiplied with these probabilities. In this way, the resulting effective interaction $U(\omega)$ only depends on the MLWFs that span the $d$ subspace and is basically independent of the used electronic structure method.

In a more formal approach, one can define $P_{d}$ as the density correlation function $P_{d}\left(\mathbf{r} t, \mathbf{r}^{\prime} t^{\prime}\right)=-i\left\langle\Psi_{0}\right| \hat{T}\left[\hat{n}_{d}(\mathbf{r} t)\right.$, $\left.\hat{n}_{d}\left(\mathbf{r}^{\prime} t^{\prime}\right)\right]\left|\Psi_{0}\right\rangle$ with the Kohn-Sham determinant $\Psi_{0}$, the timeordering operator $\hat{T}$, and the Heisenberg density operator $\hat{n}(\mathbf{r} t)=\hat{n}_{d}(\mathbf{r} t)+\hat{n}_{r}(\mathbf{r} t)$ decomposed according to the $d$ subspace and the rest. Without time-dependent external fields, $P_{d}$ only depends on the time difference $t-t^{\prime}$. A Fourier transformation then yields the Lehmann representation described above.

The ground-state calculations are carried out using the FLAPW method as implemented in the FLEUR code ${ }^{13}$ within the LDA exchange-correlation potential. ${ }^{14}$ The MLWFs are constructed with the WANNIER90 code. ${ }^{15,16}$ We include six bands per TM atom in the construction of the MLWFs, i.e., the five $d$ bands and the itinerant $s$ band. The matrix elements of the effective Coulomb potential $U$ in the MLWF basis are given by $U_{\mathbf{R} n_{1} n_{3} ; n_{4} n_{2}}^{\alpha \beta}(\omega)=$ $\iint w_{n_{1} \mathbf{R}}^{\alpha *}(\mathbf{r}) w_{n_{3} \mathbf{R}}^{\alpha}(\mathbf{r}) U\left(\mathbf{r}, \mathbf{r}^{\prime} ; \omega\right) w_{n_{4} \mathbf{R}}^{\beta *}\left(\mathbf{r}^{\prime}\right) w_{n_{2} \mathbf{R}}^{\beta}\left(\mathbf{r}^{\prime}\right) d^{3} r d^{3} r^{\prime}$. The effective Coulomb potential $U\left(\mathbf{r}, \mathbf{r}^{\prime} ; \omega\right)$ itself is calculated within the cRPA implemented in the SPEX code ${ }^{17}$ (for further technical details, see Ref. 18). We define the average on-site diagonal (direct intra-orbital) and off-diagonal (exchange interorbital) matrix elements of the effective Coulomb potential as $U=\frac{1}{5} \sum_{n}^{(d)} U_{\mathbf{R} n n ; n n}^{\alpha \beta}$ and $J=\frac{1}{20} \sum_{m, n(m \neq n)}^{(d)} U_{\mathbf{R} m n ; n m}^{\alpha \beta}$. The average off-diagonal (direct interorbital) Coulomb matrix elements are given by the relation $U^{\prime}=U-2 J$. Although the matrix elements of the effective Coulomb potential are formally spin-dependent due to the spin dependence of the MLWFs, we find that this dependence is negligible in practice.

We start with the discussion of the unscreened (bare) Coulomb interaction in the TMs. Figure 1 shows the average bare on-site direct $(V)$ Coulomb matrix elements for the $3 d$, $4 d$, and $5 d$ TM series in the NM state. In the inset, we show the results for exchange $\left(J_{b}\right)$ Coulomb matrix elements. Note that among the $3 d$ series, $\mathrm{Fe}, \mathrm{Co}$, and $\mathrm{Ni}$ are ferromagnetic (FM)

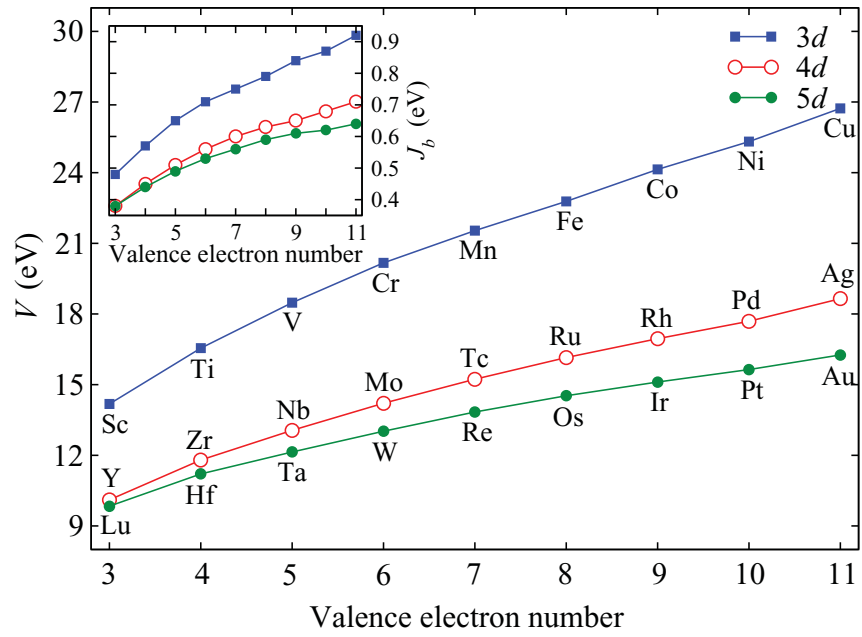

FIG. 1. (Color online) Average bare on-site direct Coulomb matrix elements between the $d$ orbitals for TMs. In the inset we show the results for exchange Coulomb matrix elements.

while $\mathrm{Cr}$ orders antiferromagnetically. Also $\mathrm{Mn}$ is FM in the bcc structure with $a=2.91 \AA$. For these elements, we find that matrix elements of the bare Coulomb potential for magnetic and NM states are nearly identical. Within each series, both $V$ and $J_{b}$ increase monotonically with the $d$ electron number. This can be explained by the fact that, as one moves from the left to the right within one row of the Periodic Table, the nuclear charge increases and causes the $d$-wave functions to contract, which gives rise to the observed trend for $V$ and $J_{b}$. On the other hand, the localization of the $d$ electrons decreases within one column of the Periodic Table from $3 d$ to $5 d$ elements. As a consequence, $V$ and $J_{b}$ decrease in the same direction. This decrease is more pronounced for late transition metals.

Efficient $s p$ screening in TMs significantly reduces the bare Coulomb interaction $V$. Calculated Hubbard $U$ and $J$ parameters for the NM state of the TMs are presented in Fig. 2. Results for the magnetic states of the $3 d$ elements are also included. For comparison, matrix elements of the fully screened Coulomb interaction $(\tilde{U}, \tilde{J})$ are given. As seen in Fig. 2, in contrast to the bare direct Coulomb interaction $V$, the Hubbard $U$ shows a nonmonotonic behavior, i.e., it increases from the early TMs and reaches a plateaulike behavior around half-filling, whereas $\tilde{U}$ is almost constant across the TM series, except for the elements with completely filled $d$ shells such as $\mathrm{Cu}$. This behavior of the Hubbard $U$ reflects a substantial contribution of the $d$ - $d$ transitions to the fully screened Coulomb interaction $\tilde{U}$, especially around half-filling. In contrast to the bare Coulomb $V$, the Hubbard $U$ parameter is very sensitive to the $d$ electron number and $d$ orbital filing. In metals we are in the strong coupling limit, $v\left|P_{r}\right| \gg 1$, and thus $U \simeq-\frac{1}{P_{r}}$. Since $P_{r}$ depends mostly on the electronic structure of the screening electrons, this explains why isovalent TMs with the same crystal structure of $4 d$ and $5 d$ exhibit very similar $U$ values but different values of $\mathrm{Mn}, \mathrm{Fe}$, and $\mathrm{Co}$, which have different crystal structures. Furthermore, by constrained NM and proper spin-polarized treatments of the magnetic elements, we show that spin polarization has a strong influence on $U$ and $\tilde{U}$. The calculated $U$ values for $\mathrm{Cr}, \mathrm{Fe}, \mathrm{Co}$, and $\mathrm{Ni}$ turn out to be larger in the magnetic state 


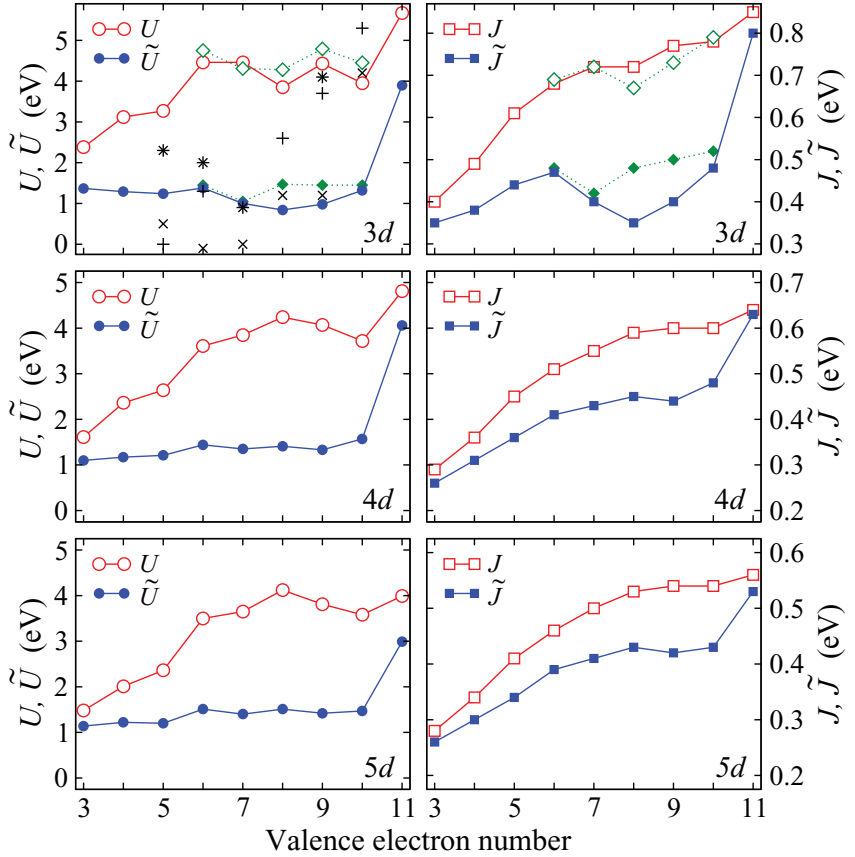

FIG. 2. (Color online) Left panels: $U$ and $\tilde{U}$ for the TM series. With open and filled diamonds we show $U$ and $\tilde{U}$ for the magnetic state of $3 d$ TMs. For comparison, experimental results from Refs. 19 (stars), 20 (pluses), and 21 (crosses) as available are given. Right panels: the same for $J$ and $\tilde{J}$.

than in the NM one, while the situation is just the opposite for Mn, which was explained in Ref. 18 for the case of $\tilde{U}$ by the different screening due to the available electrons at the Fermi energy. The same discussion holds also for partially screened $U$. Our calculated Hubbard $U$ parameters for the $3 d$ series are in good agreement with recent cRPA studies of Miyake et al. ${ }^{11}$ for late TMs as well as cLDA calculations of Nakamura et al. ${ }^{8}$ for early TMs. Experimentally, the Hubbard $U$ parameters for $3 d$ TMs are deduced from a combined use of Auger and x-ray photoemission spectroscopy. ${ }^{19-21}$ Results from three different groups are included in Fig. 2 for comparison. As seen, the experimental $U$ parameters are rather scattered. Our calculated $U$ values are in good agreement with measurements of Kaurila et al. ${ }^{19}$ as well as Yin et al. ${ }^{20}$ for late TMs. So far, we have focused only on the effective intra-orbital direct Coulomb interaction $U$. The same discussion holds also for the interorbital direct and exchange Coulomb interaction $U^{\prime}$ and $J$, respectively. Note that in contrast to $U$ and $U^{\prime}$, renormalization of the $J$ is rather small, i.e., $J$ is close to the atomic value $J_{b}$. However, the $d$ - $d$ transitions substantially reduce $\tilde{J}$, especially for late TMs. It should also be noted that while the bare $V$ has a long-range behavior, the $U$ shows much faster damping. The calculated nearest-neighbor $U$ values lie between 0.1 and $0.4 \mathrm{eV}$, being maximal for TMs with half-filled $d$ bands.

In Table I, we present the orbital dependence of the Hubbard $U$ for bcc $\mathrm{V}, \mathrm{Nb}$, and $\mathrm{Ta}$ and fcc $\mathrm{Ni}, \mathrm{Pd}$, and Pt. Figure 3 shows the frequency dependence of $U$ and $J$ for the same elements. As can be seen, the crystal structure has a sizable influence not only on the orbital anisotropy of the Coulomb matrix elements but also on the frequency dependence of $U$. For TMs with bcc structure, the effective interaction between
TABLE I. Hubbard $U$ for $e_{g}$ and $t_{2 g}$ orbitals (in $\mathrm{eV}$ ) for bcc $\mathrm{V}$, $\mathrm{Nb}$, and $\mathrm{Ta}$ and fcc $\mathrm{Ni}, \mathrm{Pd}$, and $\mathrm{Pt}$.

\begin{tabular}{lcccccc}
\hline \hline & $\mathrm{V}$ & $\mathrm{Nb}$ & $\mathrm{Ta}$ & $\mathrm{Ni}$ & $\mathrm{Pd}$ & $\mathrm{Pt}$ \\
\hline$U\left(e_{g}\right)$ & 3.47 & 2.78 & 2.58 & 4.04 & 3.76 & 3.63 \\
$U\left(t_{2 g}\right)$ & 3.13 & 2.55 & 2.21 & 3.90 & 3.69 & 3.55 \\
\hline \hline
\end{tabular}

$d$ electrons in $e_{g}$ orbitals is about $0.3 \mathrm{eV}$ larger than that in $t_{2 g}$ orbitals, whereas this difference is about $0.1 \mathrm{eV}$ for TMs having close-packed fcc and hcp (results not shown) structures. For the former TMs, the $U(\omega)$ show strong variations at low frequencies (see Fig. 3), which suggests that the use of the static value $U(\omega=0)$ in model Hamiltonians may be inappropriate. For the latter elements, $U(\omega)$ shows a smoother behavior. At the plasmon frequency $(20-30 \mathrm{eV})$ the $U(\omega)$ increases rapidly. Above this frequency, the screening is not effective and $U(\omega)$ approaches the bare value (compare Fig. 1). In contrast to $U(\omega)$, the exchange $J(\omega)$ is only weakly energy-dependent and does not show significant variations at the plasmon frequency.

Finally, we discuss the strength of the electronic correlations and the instability of the paramagnetic state toward ferromagnetism for the late $3 d$ TMs. In Fig. 4(a), we show $U / W$ ratios for the TM series, where the $d$ bandwidths $W$ are obtained from the single-particle band structure. As seen, similar to the bare Coulomb interaction $V$, the $U / W$ ratio increases from early to late TMs and, as a result, the correlation strength increases. Despite the similar $U$ parameters for isovalent TMs, the $3 d$ elements have larger $U / W$ ratios than $4 d$ and $5 d$ elements due to the much smaller bandwidths. For all TMs, except $\mathrm{Cu}$ and $\mathrm{Ag}$, we have $U / W<1$, which reveals weak electronic correlations in these materials. Ferromagnetism of the late TMs can be related to large $U / W$ ratios, but this condition is not sufficient. In addition, the dimensionality and the crystal structure, which dictates the shape of the density of states (DOS), is crucial for the appearance of itinerant ferromagnetism. ${ }^{22}$ In a mean-field treatment of itinerant ferromagnetism, the instability of the paramagnetic state is given by the Stoner criterion $\operatorname{IN}\left(\epsilon_{F}\right)>$ 1 , where $I$ is the Stoner parameter and $N\left(\epsilon_{F}\right)$ is the DOS at the Fermi level in the NM state. Using the Hartree-Fock solution of the multiorbital Hubbard model, Stollhoff et al. ${ }^{23}$ proposed a relationship between the Stoner parameter $I$ and the Hubbard $U$ and $J$, which is given by $I=(U+6 J) / 5$. These authors

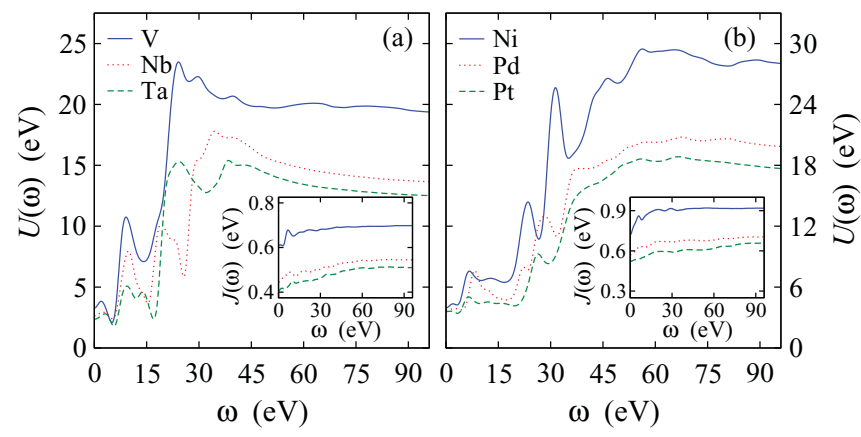

FIG. 3. (Color online) (a) $U(\omega)$ for bcc $\mathrm{V}, \mathrm{Nb}$, and $\mathrm{Ta}$. The inset shows $J(\omega)$ for the same elements. (b) The same as (a) for fcc Ni, Pd, and Pt. 


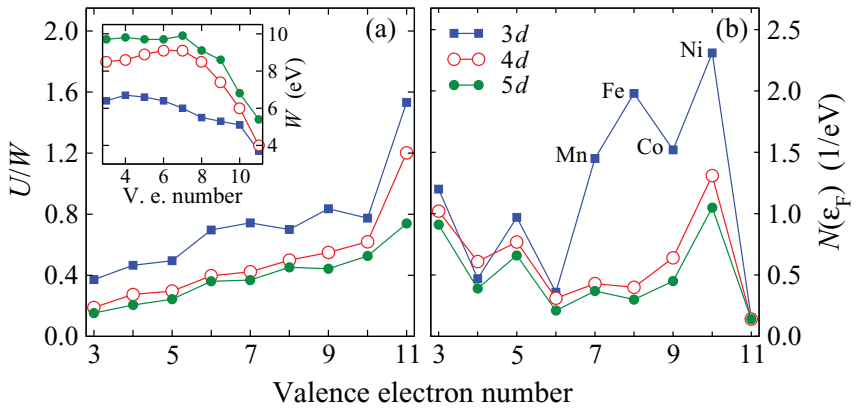

FIG. 4. (Color online) (a) The ratio $U / W$ of the effective Coulomb interaction $U$ and the $d$ bandwidth $W$ for the TM series. The inset shows the $d$ bandwidths. (b) Density of states $N\left(\epsilon_{F}\right)$ at the Fermi level for the NM states.

showed that electron correlation reduces $I$ by roughly $40 \%$. Using the calculated $U$ and $J$ values, we get $I=0.98,1.08$, and 1.04 for $\mathrm{Fe}, \mathrm{Co}$, and $\mathrm{Ni}$, respectively, which is very close to the values $0.92(\mathrm{Fe}), 0.98(\mathrm{Co})$, and $1.02(\mathrm{Ni})$ obtained from linear-response calculations. ${ }^{24}$ Among the $3 d$ series, only $\mathrm{Mn}$, $\mathrm{Fe}, \mathrm{Co}$, and Ni satisfy the Stoner criterion due to the large DOS at the Fermi level [see Fig. 4(b)], and the paramagnetic state is unstable toward the formation of ferromagnetism. The $4 d$ element $\mathrm{Pd}$ is nearly ferromagnetic. It shows strong spin fluctuations and exchange enhancement. ${ }^{25}$

In conclusion, by employing an alternative parameterfree cRPA scheme, we have calculated the effective onsite Coulomb interaction (Hubbard $U$ ) between localized $d$ electrons in TMs. We have shown that the Hubbard $U$ depends on the crystal structure, spin polarization, $d$ electron number, and $d$ orbital filling, while it is insensitive to the $d$ character of the elements. Most of the isovalent TMs assume similar $U$ values. The obtained $U$ parameters for the $3 d$ TMs are in good agreement with previous studies as well as available experimental data, and they predict correctly the paramagnetic instability toward the ferromagnetic state for the late $3 d$ 's. The $U(J)$ values as calculated in the presented approach increase considerably the predictive power of the LDA $+U$ and LDA + DMFT schemes applied to describe correlated electron materials.

Fruitful discussions with F. Freimuth, A. Schindlmayr, T. Miyake, F. Aryasetiawan, and R. Sakuma are gratefully acknowledged. This work has been supported in part by the DFG through the Research Unit FOR-1346. *e.sasioglu@fz-juelich.de

${ }^{1}$ W. Kohn and L. J. Sham, Phys. Rev. 140, A1133 (1965).

${ }^{2}$ J. Hubbard, Proc. R. Soc. London, Ser. A 276, 238 (1963).

${ }^{3}$ P. W. Anderson, Phys. Rev. 124, 41 (1961).

${ }^{4}$ A. Georges, G. Kotliar, W. Krauth, and M. Rozenberg, Rev. Mod. Phys. 68, 13 (1996)

${ }^{5}$ V. I. Anisimov, A. I. Poteryaev, M. A. Korotin, A. O. Anokhin, and G. Kotliar, J. Phys. Condens. Matter 9, 7359 (1997).

${ }^{6}$ V. I. Anisimov, J. Zaanen, and O. K. Andersen, Phys. Rev. B 44, 943 (1991).

${ }^{7}$ T. Kotani, J. Phys. Condens. Matter 12, 2413 (2000); I. Schnell,

G. Czycholl, and R. C. Albers, Phys. Rev. B 65, 075103 (2002);

I. V. Solovyev and M. Imada, ibid. 71, 045103 (2005);

M. Cococcioni and S. de Gironcoli, ibid. 71, 035105 (2005);

F. Aryasetiawan, M. Imada, A. Georges, G. Kotliar, S. Biermann, and A. I. Lichtenstein, ibid. 70, 195104 (2004).

${ }^{8}$ K. Nakamura, R. Arita, Y. Yoshimoto, and S. Tsuneyuki, Phys. Rev. B 74, 235113 (2006).

${ }^{9}$ P. H. Dederichs, S. Blügel, R. Zeller, and H. Akai, Phys. Rev. Lett. 53, 2512 (1984); V. I. Anisimov and O. Gunnarsson, Phys. Rev. B 43, 7570 (1991).

${ }^{10}$ F. Aryasetiawan, K. Karlsson, O. Jepsen, and U. Schönberger, Phys. Rev. B 74, 125106 (2006); T. Miyake and F. Aryasetiawan, ibid. 77, 085122 (2008).
${ }^{11}$ T. Miyake, F. Aryasetiawan, and M. Imada, Phys. Rev. B 80, 155134 (2009).

${ }^{12}$ N. Marzari and D. Vanderbilt, Phys. Rev. B 56, 12847 (1997).

${ }^{13}$ [http://www.flapw.de].

${ }^{14}$ J. P. Perdew and A. Zunger, Phys. Rev. B 23, 5048 (1981).

${ }^{15}$ A. A. Mostofi, J. R. Yates, Y.-S. Lee, I. Souza, D. Vanderbilt, and N. Marzari, Comput. Phys. Commun. 178, 685 (2008).

${ }^{16}$ F. Freimuth, Y. Mokrousov, D. Wortmann, S. Heinze, and S. Blügel, Phys. Rev. B 78, 035120 (2008).

${ }^{17}$ C. Friedrich, S. Blügel, and A. Schindlmayr, Phys. Rev. B. 81, 125102 (2010).

${ }^{18}$ E. Şaşığlu, A. Schindlmayr, C. Friedrich, F. Freimuth, and S. Blügel, Phys. Rev. B. 81, 054434 (2010).

${ }^{19}$ T. Kaurila, J. Väyrynen, and M. Isokallio, J. Phys. Condens. Matter 9, 6533 (1997)

${ }^{20}$ L. I. Yin, T. Tsang, and I. Adler, Phys. Rev. B 15, 2974 (1977).

${ }^{21}$ D. K. G. de Boer, C. Haas, and G. A. Sawatzky, J. Phys. F 14, 2769 (1984).

${ }^{22}$ S. Sakai, R. Arita, and H. Aoki, Phys. Rev. Lett. 99, 216402 (2007).

${ }^{23}$ G. Stollhoff, A. M. Oleś, and V. Heine, Phys. Rev. B 41, 7028 (1990).

${ }^{24} \mathrm{P}$. Mohn, Magnetism in the Solid State (Springer, Berlin, 2002).

${ }^{25}$ R. Doubble, S. M. Hayden, P. Dai, H. A. Mook, J. R. Thompson, and C. D. Frost, Phys. Rev. Lett. 105, 027207 (2010). 